\title{
Findings from the WeighWell feasibility study of post-partum weight loss: Challenges for a randomised controlled trial
}

\author{
A. M. Craigie ${ }^{1}$, R. S. Barbour ${ }^{2}$, A. S. Anderson ${ }^{1}$ and the WeighWell team \\ ${ }^{1}$ Centre for Public Health Nutrition Research, University of Dundee, Dundee DDI 9SY, UK and ${ }^{2}$ School of Nursing and \\ Midwifery, University of Dundee, 11 Airlie Place, Dundee DD1 4HJ, UK
}

Obesity poses a growing medical threat on a global scale. Pregnancy is a high risk period for excess weight gain with many retaining weight in the post-partum period ${ }^{(1)}$. Antenatal clinic data have identified a significantly greater proportion of women from areas of high deprivation to be obese ${ }^{(2)}$. Yet little is known about the response of post-partum women from socially deprived backgrounds to weight management interventions to inform health service development ${ }^{(3)}$. The WeighWell study was a scoping and feasibility study which aimed at developing and implementing a weight loss intervention for post-partum women living in areas of social deprivation, prior to undertaking a randomised controlled trial (RCT). The successes and challenges of recruiting a target of sixty women to a 3-month weight-loss study are reported here, with particular focus on user views.

Recruitment was targeted at socially disadvantaged communities of Tayside, Scotland. Recruitment strategies included poster and 'business card' distribution, newspaper advertisements, community group visits and letters of invitation to post-partum women (from Maternal Health Services). Participants were telephone screened for eligibility (6-18 months post-partum, BMI $\geq 25 \mathrm{~kg} / \mathrm{m}^{2}$, living in areas of moderate to high deprivation). Baseline assessments including physical activity monitoring, anthropometric measures, provision of a blood sample and completion of a lifestyle questionnaire were carried out before providing the British Heart Foundation booklet 'So You Want to Lose Weight for Good'. Participants were then randomised into comparison (CG) (no further guidance) or intervention groups (IG). Over 3 months the IG received monthly motivational interviewing sessions plus telephone support incorporating goal setting, weight monitoring and literature advising on low cost lifestyle changes. Follow-up assessments were carried out after the intervention period. Participant views were sought via post-study semi-structured interviews.

Of the 142 women responding, sixty-three $(44 \%)$ met the eligibility criteria and fifty-four of these (86\%) completed baseline assessments. Loss to follow-up was $24 \%$ in the IG and $39 \%$ in the CG. Potential efficacy of the intervention was indicated by a significant decrease in body weight of -1.6 (SD 2.0) $\mathrm{kg}$ in the IG compared with +0.2 (SD 2.2) $\mathrm{kg}$ in the CG over the 3 months. Key findings from post-study interviews with 13 from each group included the following:

- All participants reported their experience positively. However, many CG participants noted a feeling of disappointment about their allocation (one claiming it made her 'worse' for a short time after the allocation) despite being aware of the randomisation procedure.

- The intervention was particularly well received (format, approach and literature), with all describing it as useful. Sustained improvements to diet were reported by all (e.g. increases in fruit and vegetable intake, cooking of fresh food, and reductions to portion sizes, eating out, and intake of snacks and sugary foods). These extended, in most cases, to the wider family. Most also reported improvements to physical activity levels.

- Many CG participants reported making some improvements to their lifestyle as a result of an increased awareness following the assessment procedures and literature provided.

- Few difficulties were reported regarding baseline and follow-up assessments. Most difficulties related to the SenseWear physical activity arm band (discomfort), and a small number of questionnaire items.

The potential efficacy of the intervention over a relatively short period is encouraging and supports the need for a full RCT. However, CG disappointment over group allocation, their relatively high attrition and the lifestyle changes reported following baseline assessments raises question over adequate retention and achievement of a 'true control' in an RCT.

Funding was provided by the Medical Research Council (Ref GO701771).

1. Villamor E \& Cnattingius S (2006) Lancet 368(9542), 1164-1170.

2. Heslehurst N, Rankin J, Wilkinson JR et al. (2010) Int J Obes 34(3), 420-428.

3. National Institute for Heath and Clinical Excellence (2010) Public Health Guidance 27. London: NICE. 